\title{
Metallic Conductance at the Interface of Tri-color Titanate Superlattices
}

\author{
M. Kareev ${ }^{*}$ Yanwei Cao, Xiaoran Liu, S. Middey, D. Meyers, and J. Chakhalian \\ Department of Physics, University of Arkansas, Fayetteville, Arkansas 72701, USA
}

(Dated: November 22, 2013)

\begin{abstract}
Ultra-thin tri-color (tri-layer) titanate superlattices ([3u.c. $\mathrm{LaTiO}_{3} / 2$ u.c. $\mathrm{SrTiO}_{3} / 3$ u.c. $\left.\mathrm{YTiO}_{3}\right]$, u.c. $=$ unit cells) were grown in a layer-by-layer way on single crystal $\mathrm{TbScO}_{3}$ (110) substrates by pulsed laser deposition. High sample quality and electronic structure were characterized by the combination of in-situ photoelectron and ex-situ structure and surface morphology probes. Temperature-dependent sheet resistance indicates the presence of metallic interfaces in both [3u.c. $\mathrm{LaTiO}_{3} / 2$ u.c. $\left.\mathrm{SrTiO}_{3}\right]$ bi-layers and all the tri-color structures, whereas a [3u.c. YTiO $3 / 2$ u.c. $\mathrm{SrTiO}_{3}$ ] bi-layer shows insulating behavior. Considering that in the bulk $\mathrm{YTiO}_{3}$ is ferromagnetic below $30 \mathrm{~K}$, the tri-color titanate superlattices provide an opportunity to induce tunable spinpolarization into the two-dimensional electron gas (2DEG) with Mott carriers.
\end{abstract}

*Electronic address: mkareev@uark.edu 
Recent studies of emergent phenomena at the interfaces of transition metal oxides have attracted a tremendous interest due to the variety of novel phenomena, [1, 4] which are either hindered or not attainable in bulk materials including two dimensional electron gas (2DEG), [1, 3, 5] spin and orbital reconstruction at the superconductor/ ferromagnet interface, [6, 7] magneto-electric effects, [8, 9] magnetism in layers of non-magnetic materials, [10] metal-insulator transitions, [11] anomalous Hall effect[12, 13] to mention a few. In particularly, the appearance of a 2DEG was observed at the junction between two insulators composed of non-polar band insulator $\mathrm{SrTiO}_{3}$ and polar band insulator $\mathrm{LaAlO}_{3}$.[1, 14-18] Similar metallic interfaces have been reported in other oxide materials such as $\mathrm{LaTiO}_{3} / \mathrm{SrTiO}_{3}\left(\mathrm{LTO} / \mathrm{STO}\right.$ ), 19 26] $\mathrm{LaVO}_{3} / \mathrm{SrTiO}_{3}$, 27] $\mathrm{LaGaO}_{3} / \mathrm{SrTiO}_{3}, 28$, 28 $\mathrm{GdTiO}_{3} / \mathrm{SrTiO}_{3},\left[29\right.$ and $\mathrm{Al}_{2} \mathrm{O}_{3} / \mathrm{SrTiO}_{3}$. [30] On the other hand, since in complex oxides spin, charge and orbital degrees of freedom are strongly coupled, it would be interesting to investigate if the 2DEG can be spin-polarized (SP), [29, 31] possibly opening new opportunities for spintronics applications. 32 Recent, theoretical studies have predicted the possibility of SP-2DEG at the interfaces of $\mathrm{SrMnO}_{3} / \mathrm{LaMnO}_{3}$ and $\mathrm{LaAlO}_{3} / \mathrm{EuO}$. [33, 34] Based on this, designing artificial hetero-junctions of correlated oxides synthesised with unit-cell precision [15, 16, 19, 21] is a key step towards, these emerging physics and new nano-devices.

In this letter, we report growth and transport properties of tri-color titanate superlattices [3u.c. $\mathrm{LaTiO}_{3} / 2$ u.c. $\mathrm{SrTiO}_{3} / 3$ u.c. $\left.\mathrm{YTiO}_{3}\right]_{4}$ (simplified as $[3 \mathrm{LTO} / 2 \mathrm{STO} / 3 \mathrm{YTO}]$ ) and bi-color titanate superlattices [3u.c. LTO/2u.c. STO $]_{4}([3 \mathrm{LTO} / 2 \mathrm{STO}])$ and [3u.c. YTO/2u.c. STO $]_{4}([3 \mathrm{YTO} / 2 \mathrm{STO}])$ (see Fig. 1 (a)). For tri-color structures, from the bottom the stacking sequences were ABCBABCBA, and $\mathrm{A}=3 \mathrm{YTO}, \mathrm{B}=2 \mathrm{STO}, \mathrm{C}=3 \mathrm{LTO}$, respectively. In the bulk, $\mathrm{LaTi}^{3+} \mathrm{O}_{3}$ is a Mott insulator with $3 d^{1}$ electron configuration and G-type antiferromagnetic (AFM) ground state below $<146$ K, 35] whereas the Mott insulator $\mathrm{YTi}^{3+} \mathrm{O}_{3}$ (YTO) with $3 d^{1}$ electron configuration undergoes a ferromagnetic transition below $30 \mathrm{~K}$. [36] $\mathrm{SrTi}^{4+} \mathrm{O}_{3}$ with $3 d^{0}$ electronic configuration is a band-gap paramagnetic insulator. The high quality of surface and crystal morphology and electronic structure are confirmed by the combination of reflection-high-energy-electron-diffraction (RHEED), atomic force microscopy (AFM), X-ray diffraction (XRD), and in-situ X-ray photoemission (XPS) and Auger spectroscopies. In sharp contrast to the [3YTO/2STO] superlattice, for both [3LTO/2STO] and [3LTO/2STO/3YTO] films, DC conductance shows metallic character at all temperatures below $300 \mathrm{~K}$. By adding ferromagnetic YTO layers into the well-studied 
LTO/STO superlattice, [19 26] a new tri-color titanate superlattice $\mathrm{LaTiO}_{3} / \mathrm{SrTiO}_{3} / \mathrm{YTiO}_{3}$ (LTO/STO/YTO) is formed. In this structure, because of small thickness of the STO layer (2 and 3 u.c.), conducting carriers of 2DEG formed at the LTO/STO interface are able to interact with magnetic moments of the YTO layer thus opening a route to the spin polarized version of 2DEG.

All films in this work were grown on (110) surface (in orthorhombic notation) of $\mathrm{TbScO}_{3}$ (TSO) single crystal substrates by pulsed laser deposition using a KrF excimer laser operating at $\lambda=248 \mathrm{~nm}$ and $2 \mathrm{~Hz}$ pulse rate with $\sim 2 \mathrm{~J} / \mathrm{cm}^{2}$ fluence. TSO is selected for two reasons, (1) bulk $\mathrm{TbScO}_{3}$ is a insulator without $\mathrm{Ti}$ in its chemical formula, and (2) bulk TSO has an orthorhombic structure with lattice constant closely matching the active titanate layers (see Table I). The growth is monitored by in-situ high-pressure RHEED. During growth, the temperature of the substrate is held at $950^{\circ} \mathrm{C}$; we report heater temperature. Since oxygen deficient LTO and STO structures can easily form other chemical phases at high oxygen pressure, e.g. $\mathrm{La}_{2} \mathrm{Ti}_{2} \mathrm{O}_{7}$ and $\mathrm{Y}_{2} \mathrm{Ti}_{2} \mathrm{O}_{7},\left[36\right.$, 38] low oxygen pressure $\left(1-3 \times 10^{-6}\right.$ Torr $)$ was maintained during the deposition in this work. After the growth, all samples were cooled at about $15^{\circ} \mathrm{C} / \mathrm{min}$ rate, keeping oxygen pressure constant. Fig. 1 (c)-(e) shows a sequence of RHEED images for a tricolor superlattice [3 LTO/2 STO/3 YTO] upon completion of a specific layer in the junction. The sharp RHEED patterns with expected orthorhombic symmetry indicate the layer-by-layer two dimensional (2D) growth mode with proper epitaxial relations among the layers and the substrate. In addition, smooth surface topography of the films was confirmed by AFM; the obtained average surface roughness was found to be $<120 \mathrm{pm}$. Structural quality, tensile strain and proper epitaxy of the superlattices are further confirmed by X-ray Diffraction (XRD) using $\mathrm{Cu} \mathrm{K}_{\alpha}$ radiation shown in Fig. 1 (f). DC transport properties were measured in van der Pauw geometry by a Physical Properties Measurement System (PPMS) operating in high resolution mode.

To assure that emergent phenomena arises from the interface and not secondary chemical phases or defects, electronic structure and chemical composition of the films was further investigated by in-situ X-ray photoemission and Auger (not shown) spectroscopies; no discernable impurity signal was observed. On the other hand, as shown in Fig. 2, in three reference films 20 u.c. LTO, 20 u.c. STO, and 25 u.c. YTO (the same growth conditions as for tri-color superlattice and TSO substrate), the two main Ti peaks marked as Ti $2 \mathrm{p}_{1 / 2}$ and $\mathrm{Ti} 2 \mathrm{p}_{3 / 2}$ for $\mathrm{Ti}^{3+}$ and $\mathrm{Ti}^{4+}$ are clearly seen. The peak separation between $\mathrm{Ti}^{3+}$ and $\mathrm{Ti}^{4+}$ 
is about $2 \mathrm{eV}$, showing good agreement with previously reported data. [39] The overall lineshape of the three reference films affirms that after growth the LTO, STO, and YTO layers maintain proper bulk-like valence states of $\mathrm{Ti}$. At the same time, for [3LTO/2STO/3YTO], the features of both $\mathrm{Ti}^{3+}$ and $\mathrm{Ti}^{4+}$ are observed simultaneously as deduced from the titanium binding energy positions in the reference films.

Next we turn our attention to the transport properties of the tri-color superlattices. As shown in Fig. 1, there are two distinct interfaces separated by STO layers, namely, LTO/STO and YTO/STO. The well-studied LTO/STO interface is known to be metallic and represents a prototypical 2DEG system;[19]26] to-date, transport properties of the YTO/STO interface have not been reported. To understand the conducting behavior of the tri- and bi-color samples we first compare their transport behavior to the bulk-like reference samples of 20 u.c. LTO, 20 u.c. STO and 25 u.c. YTO grown on TSO. All those single composition layers demonstrate characteristic insulating behavior starting from 300 $\mathrm{K}$ (not shown); this result excludes the effects of defects and/or oxygen doping in LTO, STO, and YTO layers during the tri-color superlattice growth. We also point out, that this observation is particularly important for LTO layers, since in the bulk LTO can be easily oxygen over-doped to form metallic $\mathrm{LaTiO}_{3+\delta}$.40] Moreover, in Fig. 1(c) a typical RHEED pattern observed during the LTO growth is shown, yielding well-defined spots and streaks without twofold superstructure. In the case of oxygen overdoping, the appearance of such superstructure peaks is expected, since they would originate from the c-axis doubling in the perovskite unit cell, as previously reported for $\mathrm{LaTiO}_{3.5}$. [41]

After the expected conducting behavior of individual constituent layers was established, we proceed with study of the of tricolor superlattices. Figure 3 (a) demonstrates the temperature dependence of [3 LTO/2 STO/3 YTO]. As immediately seen, unlike individual layers the tri-layer structure displays metallic behavior at the wide temperature range from RT down to $2 \mathrm{~K}$. To elucidate the origin of metallicity in this structure we investigate conducting properties of individual YTO/STO and LTO/STO in the complimentary bi-color samples (Fig. 3). As seen in Fig. 3 (b) sheet resistance of the [3YTO/2STO] interface is high $\sim 30 \mathrm{k} \Omega$ at room temperature (RT) and rapidly increases with lowered temperature to $\sim 514 \mathrm{k} \Omega$ at $70 \mathrm{~K}$; this result confirms the insulating character of the YTO/STO interface. It is interesting to note that this behavior is also different from the bulk $\mathrm{Y}_{1-x} \mathrm{Ca}_{x} \mathrm{TiO}_{3}$ material which is metallic for $0.4<x<0.8$. [42, 43] In contrast to the doped bulk material, the insulating 
character of the YTO/STO interface is consistent with the theory predicted band gap for a monolayer of YO buried in STO layers. 21] Based on this result and the fact that the LTO/STO samples demonstrate distinct metallic behavior, we conclude that conductivity in the tri-color superlattice mainly originates from the LTO/STO interface. Furthermore, assuming that the conducting channels are homogenious, the averaged (a bulk-like resistivity $\rho$ (in ohm per $\mathrm{cm}$ ) can be calculated by multiplying the sheet resistance by the film thickness in $\mathrm{cm}$ ) resistivity of [3LTO/2STO] reaches $\sim 227 \mu \Omega \cdot \mathrm{cm}$ very similar to the previously reported value of $200 \mu \Omega \cdot \mathrm{cm}$ in the best [2LTO/3STO] samples.[20] In comparison to this, as shown in Fig. 3 (a), sheet resistance of tricolor films have similar temperature dependence albeit with slightly larger value of resistance. This increasing of residual resistance is expected and likely arises from the enhanced scattering of conducting carriers by the YTO/STO interface.

In summary, we have developed layer-by-layer growth of high quality tri-color and bicolor titanate superlattices. The crystal and electronic structures of the film are confirmed by XRD, AFM, XPS and Auger spectroscopies. Unlike constituent layers which are all insulators, the tri-color superlattices demonstrate distinct metallic behaviour at wide range of temperatures. A comparison of the bi-color $[3 \mathrm{YTO} / 2 \mathrm{STO}]$ and $[3 \mathrm{LTO} / 2 \mathrm{STO}]$ seems confirm that metallicity stems from the LTO/STO interface leaving YTO/STO insulating. This heterojunctions with the two dissimilar Mott/band gap interfaces may provide a another route to induce tunable spin-polarization (by controlling thickness of STO layer in tri-color structures) into 2DEG of the LTO/STO interface to form a convenient platform for novel spintronics applications. By tuning thickness of $\mathrm{SrTiO}_{3}$ layer in tri-color structures, the spin-polarization may be controlled. Retaining the intrinsic magnetic field for spin accumulation, without any gate voltage applied, 44 may enable design of spintronic devices, which could exploit the large Rashba coupling in 2DEG. 


\section{Acknowledgments}

JC is supported by DOD-ARO Grant No. 0402-17291. We deeply acknowledge discussions with Christsos Panagopolous.

[1] H. Y. Hwang, Y. Iwasa, M. Kawasaki, B. Keimer, N. Nagaosa, and Y. Tokura, Nature Mater., 11, 103 (2012).

[2] J. Chakhalian, A. J. Millis, and J. Rondinelli, Nature Mater., 11, 92 (2012).

[3] J. Mannhart, and D. G. Schlom, Science, 327, 1607 (2010).

[4] Pavlo Zubko, Sefano Gariglio, Marc Gabay, Philippe Ghosez, and Jean-Marc Triscone, Annu. Rev. Condens. Matter Phys., 2, 141-165 (2011).

[5] J. Mannhart, D. H. A. Blank, H. Y. Hwang, A. J. Millis, and J.-M. Triscone, MRS Bull., 33, 1027 (2008).

[6] J. Chakhalian, J. W. Freeland, G. Srajer, J. Strempfer, G. Khaliullin, J. C. Cezar, T. Charlton, R. Dalgliesh, C. Bernhard, G. Cristiani, H.-U. Habermeier, and B. Keimer, Nature Phys., 2, $244(2006)$.

[7] J. Chakhalian, J. W. Freeland, H.-U. Habermeier, G. Cristiani, G. Khaliullin, M. van Veenendaal, and B. Keimer, Science, 318, 1114 (2007).

[8] Ho Nyung Lee, Hans M. Christen, Matthew F. Chisholm, Christopher M. Rouleau, and Douglas H. Lowndes, Nature, 433, 395 (2005).

[9] K. Rogdakis, J. W. Seo, Z. Viskadourakis, Y. Wang, L. F. N. Ah Qune, E. Choi, J. D. Burton, E. Y. Tsymbal, J. Lee, and C. Panagopoulos, Nature Comm., 3, 1064 (2012).

[10] K. S. Takahashi, M. Kawasaki, and Y. Tokura, Appl. Phys. Lett., 79, 1324 (2001).

[11] Jian Liu, M. Kareev, D. Meyers, B. Gray, P. Ryan, J. W. Freeland, and J. Chakhalian, Phys. Rev. Lett., 109, 107402 (2012).

[12] Y. Kozuka, A. Tsukazaki, D. Maryenko, J. Falson, S. Akasaka, K. Nakahara, S. Nakamura, S. Awaji, K. Ueno, and M. Kawasaki, Phys. Rev. B, 84, 033304 (2011).

[13] H. Nakayama, M. Althammer, Y.-T. Chen, K. Uchida, Y. Kajiwara, D. Kikuchi, T. Ohtani, S. Geprägs, M. Opel, S. Takahashi, R. Gross, G. E. W. Bauer, S. T. B. Goennenwein, and E. Saitoh, Phys. Rev. Lett. 110, 206601 (2013). 
[14] A. Ohtomo, and H. Y. Hwang, Nature, 427, 423 (2004).

[15] N. Nakagawa, H. Y. Hwang, and D. A. Muller, Nature Mater. 5, 204 (2006).

[16] P. R. Wilmott, S. A. Pauli, R. Herger, C. M. Schlepütz, D. Martoccia, B. D. Patterson, B. Delley, R. Clarke, D. Kumah, C. Cionca, and Y. Yacoby, Phys. Rev. Lett. 99, 155502 (2007).

[17] S. Thiel, G. Hammerl, A. Schmehl, C. W. Schneider, and J. Mannhart, Science 313, 1942 (2006).

[18] N. Reyren, S. Thiel, A. D. Caviglia, L. Fitting Kourkoutis, G. Hammerl, C. Richter, C. W. Schneider, T.Kopp, A.-S. Rüetschi, D. Jaccard, M. Gabay, D. A. Muller, J.-M. Triscone, and J. Mannhart, Science, 317, 1196 (2007).

[19] A. Ohtomo, D. A. Muller, J. L. Grazul, and H. Y. Hwang, Nature, 419, 378 (2002).

[20] K. Shibuya, T. Ohnishi, M. Kawasaki, H. Koinuma, and M. Lippmaa, Jpn. J. Appl. Phys., 43, L1178 (2004).

[21] H. W. Jang, D. A. Felker, C. W. Bark, Y. Wang, M. K. Niranjan, C. T. Nelson, Y. Zhang, D. Su, C. M. Folkman, S. H. Baek, S. Lee, K. Janicka, Y. Zhu, X. Q. Pan, D. D. Fong, E. Y. Tsymbal, M. S. Rzchowski, and C. B. Eom, Science, 331, 886 (2011).

[22] J. S. Kim, S. S. A. Seo, M. F. Chisholm, R. K. Kremer, H.-U. Habermeier, B. Keimer, and H. N. Lee, Phys. Rev. B, 82, 201407 (2010).

[23] R. Ohtsuka, M. Matvejeff, K. Nishio, R. Takahashi, and M. Lippmaa, Appl. Phys. Lett., 96, 192111 (2010).

[24] A. Rastogi, J. J. Pulikkotil, S. Auluck, Z. Hossain, and R. C. Budhani, Phys. Rev. B, 86, $075127(2012)$.

[25] J. Biscaras, N. Bergeal, A. Kushwaha, T. Wolf, A. Rastogi, R. C. Budhani, and J. Lesueur, Nature Commun. 1, 89 (2010).

[26] J. Biscaras, N. Bergeal, S. Hurand, C. Feuillet-Palma, A. Rastogi, R. C. Budhani, M. Grilli, S. Capara, and J. Lesueur, Nature Mater. 12, 542-548 (2013).

[27] Y. Hotta, T. Susaki, and H. Y. Hwang, Phys. Rev. Lett., 99, 236805 (2007).

[28] P. Perna, D. Maccariello, M. Radovic, U. Scotti di Uccio, I. Pallecchi, M. Codda, D. Marré, C. Cantoni, J. Gazquez, M. Varela, S. J. Pennycook, and F. Miletto Granozio, Appl. Phys. Lett. 97, 152111 (2010).

[29] P. Moetakef, J. Zhang, A. Kozhanov, B. Jalan, R. Seshadri, S. J. Allen, and S. Stemmer, Appl. Phys. Lett., 98, 112110 (2011). 
[30] Y. Z. Chen, N. Bovet, F. Trier, D. V. Christensen, F. M. Qu, N. H. Andersen, T. Kasama, W. Zhang, R. Giraud, J. Dufouleur, T. S. Jespersen, J. R. Sun, A. Smith, J. Nygård, L. Lu, B. Büchner, B. G. Shen, S. Linderoth, and N. Pryds, Nature Commun. 4, 1371 (2013).

[31] N. Reyren, M. Bibes, E. Lesne, J.-M. George, C. Deranlot, S. Collin, A. Barthélémy, and H. Jaffrès, Phys. Rev. Lett. 108, 186802 (2012).

[32] I. Žutić, J. Fabian, and S. D. Sarma, Rev. Mod. Phys. 76, 323 (2004).

[33] B. R. K. Nanda and S. Satpathy, Phys. Rev. Lett. 101, 127201 (2008).

[34] Yong Wang, Manish K. Niranjan, J. D. Burton, Joonhee M. An, Kirill D. Belashchenko, and Evgeny Y. Tsymbal, Phys. Rev. B 79, 212408 (2009).

[35] M. Cwik, T. Lorenz, J. Baier, R. Müller, G. André, F. Bourée, F. Lichtenberg, A. Freimuth, R. Schmitz, E. Müller-Hartmann, and M. Braden, Phys. Rev. B 68, 060401 (2003).

[36] S. C. Chae, Y. J. Chang, S. S. A. Seo, T. W. Noh, D.-W. Kim, and C. U. Jung, Appl. Phys. Lett. 89, 182512 (2006).

[37] J. Schubert, O. Trithaveesak, A. Petraru, C. L. Jia, R. Uecker, P. Reiche, and D. G. Schlom, Appl. Phys. Lett. 82, 3460 (2003).

[38] A. Ohtomo, D. A. Muller, J. L. Grazul, and H. Y. Hwang, Appl. Phys. Lett. 80, 3922 (2002).

[39] G. Drera, G. Salvinelli, A. Brinkman, M. Huijben, G. Koster, H. Hilgenkamp, G. Rijinders, D. Visentin, and L. Sangaletti, Phys. Rev. B 87, 075435 (2013).

[40] Y. Taguchi, T. Okuda, M. Ohashi, C. Murayama, N. Môri, Y. Iye, and Y. Tokura, Phys. Rev. B 59, 7917 (1999).

[41] J.W. Seo, J. Fompeyrine, H Siegwart, and J.-P. Locquet, Phys. Rev. B 63, 205401 (2001).

[42] Y. Taguchi, T. Tokura, T. Arima, and F. Inada, Phys. Rev. B 48, 511 (1993).

[43] K. Morikawa, T. Mizokawa, A. Fujimori, Y. Taguchi, and Y. Tokura, Phys. Rev. B 54, 8446 (1996).

[44] J. Inoue, and H. Ohno, Science 309, 2004 (2005). 


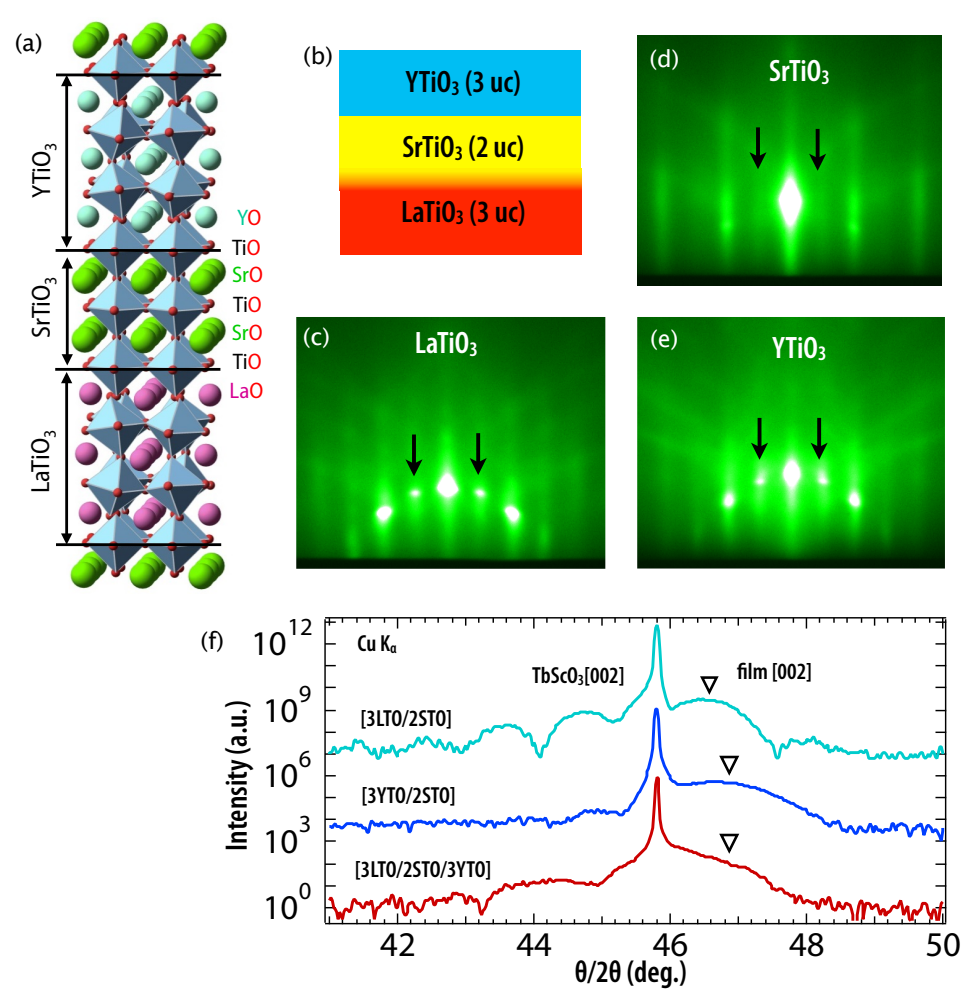

FIG. 1: (Color online) (a) and (b) possible crystal structures and block sketch of growth sequences of tricolor titanate superlattices, respectively. (c)-(e) RHEED patterns for $\mathrm{LaTiO}_{3}, \mathrm{SrTiO}_{3}$, and $\mathrm{YTiO}_{3}$ layers, respectively, during growth. Black arrows indicate half-order-peaks. (f) XRD data of bi-color film ([3LTO/2STO] and [3YTO/2STO]) and tri-color film ([3LTO/2STO/3YTO]) near [002] rod (pseudocubic structure). The main substrate peak $\left(2 \theta \sim 45.8^{\circ}\right)$ corresponds to $\sim 3.958 \AA$. The black triangles suggest broad peak of film. Since the lattice parameter of bulk STO and YTO are smaller than that of substrate (see Table I), the superlattices should be under tensile strain. For all three films, the thickness fringes were distinct, additionally testifying for flatness of the layers. 


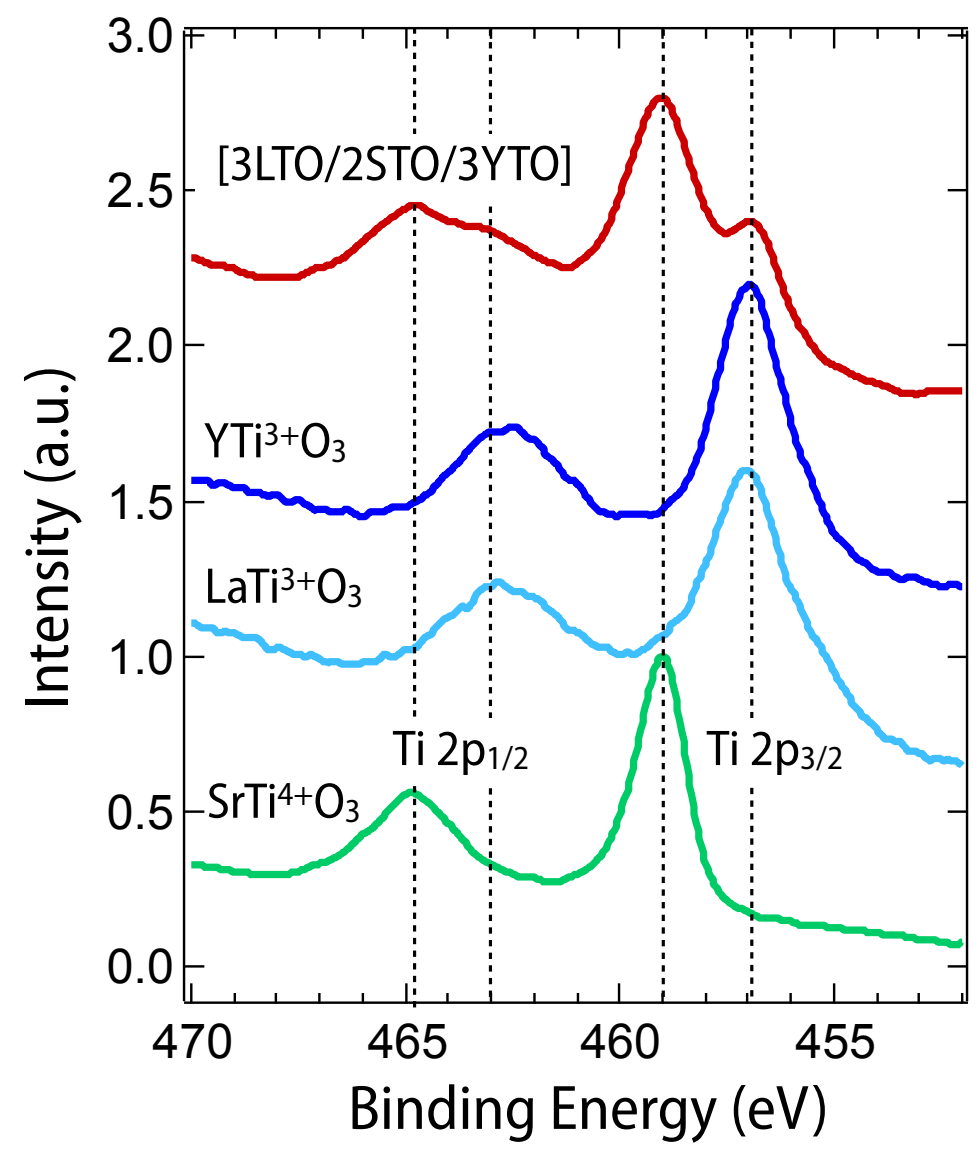

FIG. 2: (Color online) XPS spectra (Mg anode) of films with pure $\mathrm{Ti}^{4+}$ (20 uc STO), pure $\mathrm{Ti}^{3+}$ (20 uc LTO and 25 uc YTO), and mixed $\mathrm{Ti}^{4+} / \mathrm{Ti}^{3+}$ ([3LTO/2STO/3YTO $]$ ), respectively. 

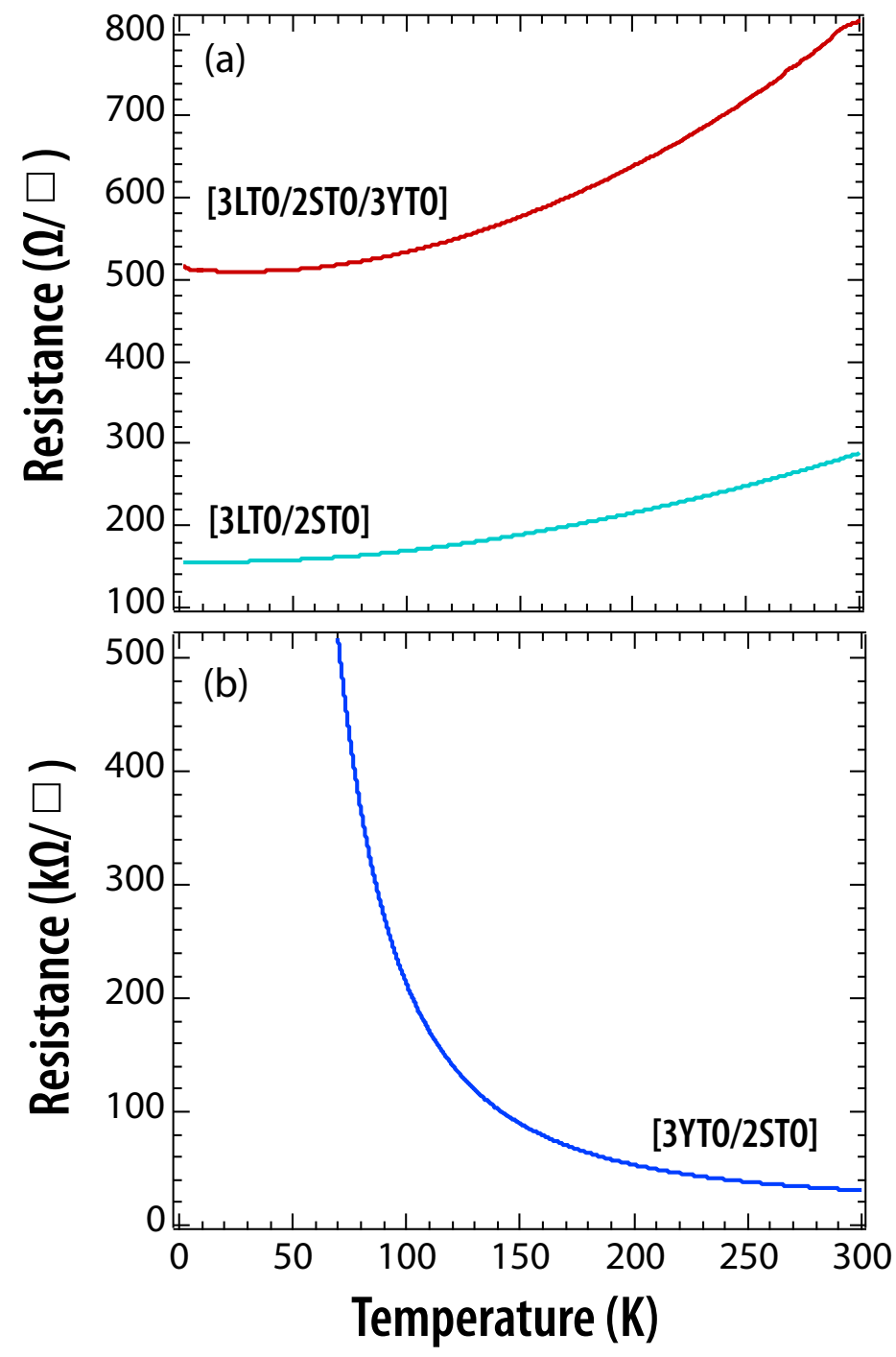

FIG. 3: (Color online) Sheet resistance of different titanate superlattices. (a) Metallic bi-color film $[3 \mathrm{LTO} / 2 \mathrm{STO}]$ and tri-color films $[3 \mathrm{LTO} / 2 \mathrm{STO} / 3 \mathrm{YTO}]$, respectively. (b) Insulated bi-color film $[3 \mathrm{YTO} / 2 \mathrm{STO}]$. 
TABLE I: Lattice parameters of four perovskite materials used in this work. a, b, c are orthorhombic or cubic lattice parameters, [21, 37] values of $\sqrt{a^{2}+b^{2}} / 2$ and $\mathrm{c} / 2$ were listed.

\begin{tabular}{lllccc}
\hline \hline Materials & $\mathrm{a}(\AA)$ & $\mathrm{b}(\AA)$ & $\mathrm{c}(\AA)$ & $\sqrt{a^{2}+b^{2}} / 2(\AA)$ & $\mathrm{c} / 2(\AA)$ \\
\hline $\mathrm{TbScO}_{3}$ & 5.466 & 5.727 & 7.915 & 3.958 & 3.958 \\
$\mathrm{LaTiO}_{3}$ & 5.595 & 5.604 & 7.906 & 3.959 & 3.953 \\
$\mathrm{YTiO}_{3}$ & 5.341 & 5.686 & 7.621 & 3.901 & 3.811 \\
$\mathrm{SrTiO}_{3}$ & 3.905 & & & 3.905 & \\
\hline \hline
\end{tabular}

